\title{
A THEOREM ON FILTERED LIE ALGEBRAS AND ITS APPLICATIONS
}

\author{
BY SHOSHICHI KOBAYASHI AND TADASHI NAGANO ${ }^{1}$
}

Communicated by S. S. Chern, January 15, 1964

The purpose of this note is to announce the following theorem and indicate a few of its applications to the classification of irreducible (complex or real) Lie algebras of linear endomorphisms of finite type of order 2 (or higher) as well as some questions on transformation groups acting on symmetric spaces. Other applications to homogeneous spaces as well as the proofs of all the results stated below will be published elsewhere.

THEOREM 1. Let L be a Lie algebra of finite dimension over a field of characteristic zero and $A$ a group of automorphisms of $L$ satisfying the following conditions:

(a) There is a decreasing sequence of A-invariant subalgebras $L=L_{-1} \supset L_{0} \supset L_{1} \supset \cdots \supset L_{k}$ such that

$$
\left[L_{p}, L_{q}\right] \subset L_{p+q} \text { for } p, q=-1,0,1, \cdots, k,
$$

where, by convention, $L_{-2}=L$ and $L_{k+1}=L_{k+2}=\cdots=0$.

(b) For every $t \in L_{p}, p \geqq 0$, such that $t \in L_{p+1}$, there is an element $x \in L$ such that $[t, x] \notin L_{p}$ (although it lies in $L_{p-1}$ by (a)).

(c) $L_{1} \neq 0$.

(d) If $S$ is an $A$-invariant subspace of $L$ containing $L_{0}$ such that $\left[L_{0}, S\right] \subset S$, then either $S=L$ or $S=L_{0}$.

Then we have

(1) $L_{2}=L_{3}=\cdots=L_{k}=0$.

(2) $L=J+\cdots+J$, where $J$ is a simple Lie algebra.

(3) L can be decomposed as follows:

$$
L=F_{-1}+F_{0}+F_{1} \text { (vector space direct sum), }
$$

in such a way that

$$
\begin{gathered}
L_{1}=F_{1}, \quad L_{0}=F_{0}+F_{1}, \\
{\left[F_{-1}, F_{-1}\right]=0, \quad\left[F_{-1}, F_{0}\right] \subset F_{-1}, \quad\left[F_{-1}, F_{1}\right] \subset F_{0},} \\
{\left[F_{0}, F_{0}\right] \subset F_{0}, \quad\left[F_{0}, F_{1}\right] \subset F_{1}, \quad\left[F_{1}, F_{1}\right]=0 .}
\end{gathered}
$$

1 Both authors are supported by NSF Grant GP-812. 
(4) There is an element, say e, in the center of $F_{0}$ such that

$$
\begin{array}{ll}
{[e, x]=-x} & \text { for } x \in F_{-1}, \\
{[e, y]=0} & \text { for } y \in F_{0}, \\
{[e, z]=z} & \text { for } z \in F_{1} .
\end{array}
$$

If the field is that of real numbers or complex numbers and if $A$ is contained in the largest connected group of automorphisms of $L$, then $L$ is a noncompact simple Lie algebra and we are able to determine $F_{-1}, F_{0}, F_{1}$ and $e$ explicitly.

In his paper $[1$, p. 159] E. Cartan listed, without proof, all irreducible complex linear groups of infinite type and also of finite type with nonvanishing "derived group" ("groupe déduit" in Cartan's terminology). Our classification using Theorem 1 shows that there are two more irreducible complex linear groups with nonvanishing derived group. Geometrically, they can be described as follows.

(1) The linear isotropy group of the group of all holomorphic transformations of the compact hermitian symmetric space $E_{6} / S O(10) \times T^{1}$.

(2) The linear isotropy group of the group of all holomorphic transformations of the compact hermitian symmetric space $E_{7} / E_{0}$ $\times T^{1}$.

Matsushima [2] gave a list of all irreducible real linear groups of infinite type as well as of finite type with nonvanishing derived group. Our classification shows that the following should be added to his list. (It should be remarked that (3)-(6) are essentially not new because their complexifications coincide with the complexifications of four groups of finite type in Matsushima's list. The complexifications of (7) and (8) (resp. (9) and (10)) coincide with the sum of two copies of (1) (resp. (2)) above.)

(3) $\rho: S U^{*}(2 p) \times S U^{*}(2 q) \times R^{*} \rightarrow G L(4 p q ; R)$. The representation $\rho$ may be described as follows. Let $a \in S U^{*}(2 p)$ and $b \in S U^{*}(2 q)$ be given by matrices in the usual manner. Let $M(2 p, 2 q ; \boldsymbol{C})$ denote the space of all $(2 p \times 2 q)$ complex matrices. Then the representation $\tilde{\rho}$ defined by

$$
\begin{array}{r}
(\tilde{\rho}(a, b, r)) u=r a u b^{-1}, \quad(a, b, r) \in S U^{*}(2 p) \times S U^{*}(2 q) \times R^{*}, \\
u \in M(2 p, 2 q ; C)
\end{array}
$$

is reducible over the reals and splits into two mutually equivalent irreducible representations, $\tilde{\rho}=\rho+\rho$. by

(4) $\rho: S L(n ; C) \times R^{*} \rightarrow G L\left(n^{2} ; R\right)$. The representation $\rho$ is defined 


$$
(\rho(a, r)) u=\operatorname{rau}^{t} \bar{a}, \quad(a, r) \in S L(n ; C) \times R^{*}, \quad u \in \mathfrak{u}(n),
$$

where the representation space $\mathfrak{u}(n)$ is the space of skew-hermitian matrices of order $n$.

(5) and (6). $\rho_{1}: S U^{*}(2 n) \times R^{*} \rightarrow G L(n(2 n+1) ; R)$ and $\rho_{2}: S U^{*}(2 n)$ $\times R^{*} \rightarrow G L(n(2 n-1) ; R)$. The representation $\tilde{\rho}$ defined by

$$
(\tilde{\rho}(a, r)) u=\operatorname{rau}^{t} \bar{a},(a, r) \in S U^{*}(2 n) \times \mathrm{R}^{*}, u \in \mathfrak{t}(2 n),
$$

splits into two irreducible representations $\rho_{1}$ and $\rho_{2}$.

(7) $\rho: S O^{5}(10) \times R^{*} \rightarrow G L(16 ; R)$. This appears as the linear isotropy group of $E_{6}^{1}$ acting on the compact symmetric space $S p(4) /(S p(2) \times S p(2))$.

(8) $\rho: S O^{1}(10) \times R^{*} \rightarrow G L(16 ; R)$. This appears as the linear isotropy group of $E_{6}^{4}$ acting on the compact symmetric space $F_{4} / S O(9)$.

(9) $\rho: E_{6}^{1} \times R^{*} \rightarrow G L(27 ; R)$. This appears as the linear isotropy group of $E_{7}^{1}$ acting on the compact symmetric space $S U(8) / S p(4)$.

(10) $\rho: E_{6}^{4} \times R^{*} \rightarrow G L(27 ; R)$. This appears as the linear isotropy group of $E_{7}^{3}$ acting on the compact symmetric space $\left(E_{6} / F_{4}\right) \times T^{1}$.

Theorem 1 recovers results of Nagano [3] on transformation groups of compact symmetric spaces (at least, when the spaces are irreducible) and, moreover, gives new information on transformation groups of noncompact symmetric spaces. We list here two typical results.

THEOREM 2. If $M$ is a noncompact irreducible Riemannian symmetric space, then no Lie group acting effectively on $M$ contains the group of isometries of $M$ as a proper subgroup.

THEOREM 3. If $M$ is a noncompact irreducible hermitian symmetric space and if a Lie group $G$ acting effectively on $M$ contains the largest connected group of isometries of $M$, then $G$ is contained in the group of isometries of $M$.

\section{BIBLIOGRAPHY}

1. E. Cartan, Les groupes de transformations continus, infinis, simples, Ann. Ecole Norm. Sup. 26 (1906), 93-161.

2. Y. Matsushima, Sur les algèbres de Lie linéaires semi-involutives, Colloque de topologie de Strasbourg, 1954, pp. 1-17.

3. T. Nagano, Transformation groups on compact symmetric spaces (to appear).

University of California, Berkeley and

TOKYo University, TOKYo, JaPan 